\title{
Modelo predictivo de la intención de adopción de Blended learning en profesores universitarios*
}

\author{
Predictive Model of the Intention to Adopt \\ Blended Learning in a University Setting
}

Recibido: enero 21 de 2013 | Revisado: junio 20 de 2013 | Aceptado: julio 21 de 2013

\author{
Antonio Víctor Martín García ** \\ MARÍA CRUZ SÁNCHez GÓMEZ *** \\ Universidad de Salamanca, España
}

doi:10.11144/Javeriana.UPSY13-2.mpia

Para citar este artículo: Martín, A. V. \& Sánchez, M. C. (2014). Modelo predictivo de la intención de adopción de Blended learning en profesores univeristarios. Universitas Psychologica, 13(2). doi:10.11144/ Javeriana.UPSY13-2.mpia

* Agradecimientos: Este trabajo ha sido desarrollado en el marco del proyecto I+D+i de Ref. EDU2010. 21299, financiado por el Ministerio de Economía y Competitividad de España. Plan Nacional I+D+i.

** Profesor Titular de la Facultad de Educación. Universidad de Salamanca. Director del Máster Universitario en Estudios avanzados de educación en la sociedad global. Correo electrónico: avmg@usal.es

**** Profesora Titular del Departamento D.O.E. y Métodos de Investigación. Facultad de Educación. Universidad de Salamanca. Correo electrónico: mcsago@usal.es

\section{RESUMEN}

El objetivo de este estudio es analizar la intención de uso de la modalidad instructiva blended learning por parte de profesores universitarios, a partir del diseño de un modelo predictivo, tomando como aportes teóricos la Teoría del Comportamiento Planificado (Theory of Planned Behavior), los modelos de aceptación tecnológica (TAM) y la Teoría de la Difusión de Innovaciones (Innovation Diffussion Theory). El modelo teórico planteado está constituido por seis constructos: Utilidad Percibida, Facilidad percibida de Uso, Compatibilidad, Imagen Social, Norma o Influencia Social e Intención de Uso. En el estudio participaron 486 profesores de diferentes universidades españolas. Para el tratamiento de los datos se realizaron Análisis Factorial Confirmatorio (AFC) y análisis causal, conforme al enfoque de ecuaciones estructurales (SEM) con estimación de parámetros, utilizando el método de Máxima Verosimilitud (ML) para el contraste de las relaciones estructurales propuestas. Los resultados revelan que los factores del modelo explican el $41 \%$ de la variabilidad de la intención de uso de blended learning en los profesores. Esta intención de adoptar b-learning está positivamente afectada por dos determinantes principales, de un lado, la utilidad percibida sobre la mejora de la práctica docente (valor estandarizado $=0.54, p<0.001$ ) y la existencia de condiciones favorables (recursos y equipamientos técnicos, humanos, etc.) de la propia universidad para su implementación $(\beta=0.26, p<0.001)$. Palabras clave autores

Modelo TAM, Teoría del Comportamiento Planificado, utilidad percibida, facilidad de uso percibida: modelos SEM, blended learning.

Palabras clave descriptors:

\section{A B S T R A C T}

The objective of this study is to analyze the intention of the use of the instructional modality blended learning to be used by academics as a predictive model using theoretical contributions from the Theory of Planned Behavior (Theory of Planned Behavior), models of acceptance technology (TAM) and the theory of diffusion of innovation (Innovation diffusion Theory). The theoretical model proposed consists of six constructs: Perceived Usefulness, Perceived Ease of Use, Compatibility Social Image, Social Influence and Intended Use. The study included 486 teachers 
from different Spanish universities. The data was analyzed using confirmatory factor analysis (CFA). Causal analysis was also utilized, conforming to structural equation approach (SEM), with parameter estimations using the maximum likelihood method (ML) for the contrast of the proposed structural relationships. The results reveal that the model factors explained $41 \%$ of the variability of the intended use of blended learning. The intention to adopt b-learning is positively affected by two main determinants, on one side the perceived usefulness of improving teaching practice (standardized value $=0.54, p<0.001$ ) and on the other the existence of favorable conditions (resources and technical equipment, human, etc.) of the university for its implementation $(\beta=0.26, p<0.001)$.

Key words authors

TAM Model, Theory of Planned Behavior, perceived usefulness, perceived ease of use: SEM models; blended learning.

Key words plus:

\section{Introducción}

Las universidades afrontan nuevos retos derivados de la integración de la era digital en el proceso de formación de sus estudiantes; esto implica la demanda de aprendizajes dinámicos, el desarrollo de nuevas competencias, de nuevos lenguajes, espacios y tiempos que obligan a la yuxtaposición de herramientas virtuales y sistemas tradicionales de enseñanza. En este contexto, surgen los modelos combinados de enseñanza denominados Blended learning, que tratan de combinar o integrar la enseñanza tradicional cara-a-cara con actividades online, sobre los que existe una amplia literatura reciente (p. ej., Bersin, 2004; Bonk \& Graham, 2006; Garrison \& Vaughan, 2008; Gerbic, 2009; Kitchenham, 2011; Wang, Fong \& Kwan, 2009). Estos modelos, derivados de los entornos empresariales y corporativos, plantean a las universidades la exigencia de un cambio de enfoque en los procesos instructivos, lo que implica la toma de decisiones y un mayor liderazgo en formas de innovación pedagógica para dar respuesta a las nuevas competencias metacognitivas requeridas hoy por las empresas. En este marco, resulta fundamental conocer los factores asociados al proceso de desarrollo e implementación de la modalidad Blended learning en la educación superior, considerando a los profesores como uno de los actores fundamentales de este proceso. Partiendo de esta idea y entendiendo por innovación la introducción de una novedad en un sistema o proceso (Rogers, 1983), se pretende analizar en qué medida es posible explicar ("modelizar") la intención de adopción o uso de este tipo de innovación educativa por parte de profesores universitarios en su práctica docente habitual.

\section{Los modelos de intención-decisión en la adopción de innovaciones}

La revisión de la literatura científica sobre el tema de las innovaciones distingue dos marcos teóricos principales que tratan de identificar y explicitar diferentes elementos conceptuales que sirven para conocer el modo como individuos, grupos corporativos o sociales adoptan y difunden una innovación dada. Se trata, por un lado, de los Modelos de Aceptación Tecnológica conocidos por sus siglas TAM (Technology Acceptance Model) y, por otro, construcciones teóricas asociadas a la denominada Teoría de Difusión de la Innovación (Innovation Diffussion Theory [IDT]). Los modelos de aceptación hacen referencia al momento en que un individuo toma la decisión de aceptar una innovación determinada. Por su parte, los de difusión se refieren al proceso mediante el cual se propaga esa innovación en el marco de un sistema social dado (ya sea este una empresa, un determinado grupo de compradores o una institución universitaria), por medio de determinados canales y a lo largo de un periodo de tiempo. Las teorías de aceptación se basan en supuestos de decisión comportamental centrados en aspectos cognitivos que van desde las creencias de un sujeto sobre una tecnología o innovación determinada hasta el comportamiento final respecto a la misma (p. ej., Davis, Bagozzi \& Warshaw, 1989; Venkatesh \& Davis, 2000; Venkatesh, Morris, Davis, Davis, 2003). Este grupo de modelos actitudinales se asientan con fuerza a mediados de la década de los 70, ofreciendo una base firme para explicar el comportamiento individual, con el plan- 
teamiento de que la adopción de una tecnología o de una innovación incorpora parámetros de tipo subjetivo, tales como creencias y actitud hacia la innovación, percepción de la influencia social o la propia intención de adoptar. La base teórica inicial de todos ellos está en la Teoría de la Acción Razonada (Theory of Reasoned Action [TAR]) formulada por Fishbein y Ajzen (1975), Azjen y Fishbein (1980) y la posterior modificación y adaptación de esta conocida como Modelo de Comportamiento Planeado (Theory of Planned Behavior [TPC]) propuesta por Azjen (1991). Los constructos más significativos de ambas han sido aplicados e integrados en nuevos modelos que tratan de buscar mayor fortaleza empírica, en particular en lo que afecta a una mejor validez predictiva y mayor consistencia psicométrica de las variables que forman parte de los mismos.

Sobre la base de estas teorías, el TAM, propuesto por Davis (1986) y por Davis, Bagozzi y Warshaw (1989), es el más ampliamente utilizado y el que cuenta con mayor apoyo empírico para explicar la aceptación de una innovación tecnológica (Venkatesh et al., 2003). El Modelo TAM sugiere que la aceptación y uso de una tecnología está fundamentalmente determinada por dos tipos de creencias: la utilidad percibida (Perceived Usefulness) y la percepción de facilidad de uso (Perceived Ease of Use). Davis et al. (1989) definen la Utilidad Percibida (UP) como aquella creencia por la cual una persona considera que el uso de un sistema particular mejorará su rendimiento profesional. Se trata de la misma idea planteada por Bandura (1982) en torno a la noción de expectativas de resultados o consecuencias de los actos, e intenta medir el grado en el que se cree que un comportamiento está vinculado a la obtención de un resultado o una recompensa laboral. Por su parte, la variable Facilidad de Uso Percibida (FUP) de un sistema o innovación puede definirse como la creencia por la que una persona considera que el uso de un sistema (tecnología, innovación, etc.) está libre de esfuerzo. También aquí se aprecia claramente la relación con el concepto de autoeficacia de Bandura (1982). Davis (1989) define este concepto como 'el grado en el que el usuario espera que el manejo de un determinado sistema conlleve la realización de menores esfuerzos'.
El efecto positivo de estas variables, en particular sobre la adopción de diversos tipos de innovaciones tecnológicas, ha sido explicado y probado empíricamente en numerosas ocasiones (Atkinson \& Kydd, 1997; Davis, 1989; Davis et al., 1989; Igbaria, Livari Maragahh, 1995; Lenderer, Maupin, Sena, \& Zhuang, 2000; Ong, YuLai \&Wang, 2004; Taylor \& Tood, 1995; Venkatesh \& Davis, 2000; Venkatesh et al., 2003). En el contexto del elearning y blearning esta relación causal ha sido también evaluada en diversos y numerosos estudios (Lee, 2006; Lee, Cheung \& Chen, 2005; Lin \& Wang, 2012; Selim, 2003; Yi \& Hwang, 2003).

Las revisiones del modelo TAM han propuesto en los últimos años modificaciones que tratan de extender y valorar la incorporación de nuevas variables al modelo. Una de las más importantes tiene que ver con la eliminación de la variable actitud como moduladora del efecto de las creencias sobre la intención de uso (Bagozzi, Davis \& Warshaw, 1992; Venkatesh \& Davis, 2000), cambio que resulta consistente con la mayoría de la investigación posterior relacionada con los modelos TAM (Gefen, Karahanna \& Straub, 2003) y supone una diferencia significativa respecto a la Teoría de la Acción Razonada, donde las creencias solo impactan a las actitudes. En este nuevo planteamiento, denominado como modelo extendido TAM2 (Venkatesh \& Davis, 2000) se incorporan como variables los determinantes de la Utilidad Percibida y de la Facilidad Percibida de Uso, como por ejemplo la Influencia Social, las Diferencias individuales, la Voluntariedad de la conducta, la Relevancia del trabajo, la Calidad de salida, la Demostrabilidad del resultado, etc. Más recientemente, la línea de investigación sobre modelos de adopción se ha visto ampliada al establecer conexiones tentativas con las teorías sobre la difusión de las innovaciones (Rogers, 1995, 2009). Uno de los ejemplos más claros de esta integración es la denominada Unified Theory of Acceptance and Use of Technology (UTAUT) propuesta por Venkatesh (Venkatesh et al., 2003; Venkatesh \& Bala, 2008), que trata de integrar aspectos conceptuales básicos de los modelos TAR, TPC, TAM y el modelo IDT de Evans Rogers. 
El modelo UTAUT reduce en cuatro variables antecedentes la intención de adopción de una innovación tecnológica: la expectativa de desempeño, la expectativa de esfuerzo, la influencia social y las condiciones facilitadoras. La Expectativa de Desempeño se define como el grado en que un individuo percibe que el uso de una innovación o de una tecnología mejorará su actividad profesional. Como fácilmente puede apreciarse, la idea es la misma que la expresada en la Utilidad Percibida (también son de uso común términos como ventaja relativa, expectativas de resultados, motivación intrínseca, etc.). Por su parte, la Expectativa de Esfuerzo coincide con la idea de facilidad de uso percibido. La Influencia Social equivale a la Norma Subjetiva del modelo TRA, sobre el grado en que un individuo cree que personas significativas para él piensan que debería usar o no un sistema, innovación o una tecnología determinada. Por último, las Condiciones Facilitadoras (facilitating conditions) se refieren al grado en que un individuo percibe que en su contexto profesional se dan las condiciones organizativas y técnicas necesarias para apoyar el uso de la tecnología. Junto a estas variables, el modelo identifica otras cuatro relacionadas con las características específicas de cada sujeto: edad, género, experiencia y voluntariedad de uso.

Revisiones más recientes del modelo ([TAM3]; Venkatesh \& Bala, 2008) conectan decididamente con la perspectiva de los modelos de difusión de la innovación (IDT), donde se introducen no solo los aspectos cognitivos del sujeto (individuales), en los que se fundamentan los modelos de aceptación tecnológica, sino también atributos de las organizaciones, las características de los sistemas organizativos, así como aspectos contextuales o situacionales. De este modo, se valora la incorporación y el peso relativo de elementos relacionados con los rasgos de personalidad y las características sociodemográficas, así como también diferentes tipos de creencias internas, como la experiencia previa del sujeto en un ámbito determinado, los hábitos personales, las rutinas y valores arraigados que pueden influir en la configuración de las actitudes y, consecuentemente, la intención y comportamiento hacia una innovación determinada. Además de esto, se evalúan elementos externos como los factores contextuales, influencia del sistema y otro tipo de variables no contempladas inicialmente en el modelo.

Fruto de esta línea de trabajo, se han ido probando y evaluando factores nuevos que se espera aporten mejores ajustes a los modelos propuestos anteriormente. Por ejemplo, dos de los constructos que más atención han recibido en los últimos años son los de Imagen Social, definida como 'el grado en que el uso de una tecnología o innovación es percibida por un individuo para mejorar su propia imagen profesional o su estatus en el contexto de la organización a la que pertenece' y, especialmente, el concepto de Compatibilidad, entendiendo por tal 'el grado en que una innovación es percibida como consistente con los valores existentes, las experiencias pasadas y las necesidades del potencial adoptante'. Por ejemplo, Karahanna, Agarwal y Angst (2006) desglosan el contenido de la compatibilidad en cuatro construcciones distintas y separables: la compatibilidad con el estilo de trabajo preferido, la compatibilidad con las prácticas de trabajo existentes, la compatibilidad con la experiencia previa y la compatibilidad con los valores propios del sistema, tratando de demostrar empíricamente las relaciones directas con la Utilidad Percibida y la Facilidad de Uso.

\section{Formulación del modelo teórico: hipótesis de investigación}

En este trabajo se considera el entorno universitario como un sistema corporativo particular, en el que los profesores constituyen uno de sus elementos clave. El objetivo es "modelizar" el uso o adopción de una innovación educativa específica, que entendemos en términos de BLearning, a partir del análisis de los determinantes que influyen en la intención de los profesores de implementarla en su práctica docente habitual. El modelo teórico definido, con base en la literatura revisada, plantea la existencia de tres variables predictoras directas de la Intención de Uso (b-learning -ICBl): Utilidad Percibida (UP), Facilidad de Uso Percibida (FUP), Condiciones Facilitadoras (FC) y dos variables Imagen Social (ISO) y Norma Social (NS) que influyen indirectamente en la intención a través de la UP. 


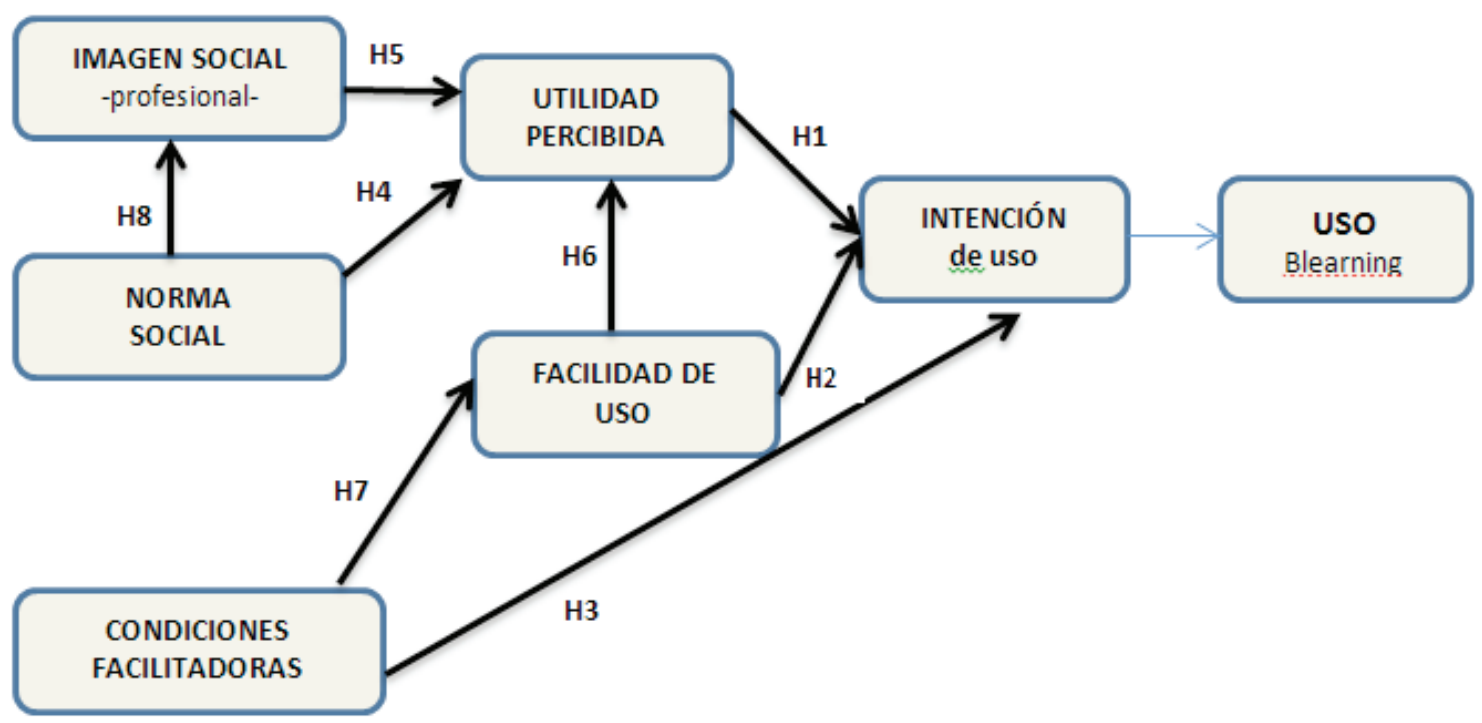

Gráfico 1. Modelo teórico TAM propuesto sobre adopción de la Innovación BLearning.

Nota. Aunque se incluye en el diagrama la conducta final (Uso Blearning) no es objeto de medida. Fuente: elaboración propia.

Se plantean las siguientes hipótesis de investigación:

- H1: La percepción de utilidad de realizar la innovación (modalidad b-learning) influye positiva y significativamente sobre la intención de usarla para la formación universitaria

- H2: La percepción de facilidad frente a complejidad de uso de la modalidad b-learning influye positiva y significativamente sobre la intención de usarla en la formación universitaria.

- H3: La percepción de condiciones facilitadoras (recursos técnicos, infraestructuras, soporte institucional, ...) en el contexto de la implementación de la modalidad formativa b-learning influye positiva y significativamente sobre la intención de usar ésta en la formación universitaria.

- H4: La Influencia o Norma social (entorno profesional docente: responsables académicos, compañeros y alumnos) influye positiva y significativamente en la percepción de utilidad de usar la modalidad formativa b-learning.

- H5: La percepción del docente de una mejora en su propia Imagen profesional influye positiva y significativamente en la utilidad percibida de implementar el entorno de formación b-learning.

- H6.- La percepción de facilidad de uso del entorno de formación b-learning influye positiva y significativamente en la utilidad percibida de implementarlo.

- H7: La percepción de Condiciones Facilitadoras del sistema está positivamente relacionada con la facilidad del uso de b-learning.

- H8: La Influencia o Norma social (entorno profesional) está positivamente relacionada con la imagen profesional del docente.

\section{Método}

\section{Procedimiento de muestreo y} características de la muestra

Para la recogida de datos se realizó un muestreo estratificado por conglomerados, con selección de las unidades primarias de muestreo (universidades) y de las unidades secundarias (facultades) de forma aleatoria proporcional, y de las unidades últimas (individuos) por rutas aleatorias y cuotas 
TABLA 1

Perfil de la muestra de profesores universitarios

\begin{tabular}{llll}
\hline Variables & categorías & $n$ & $\%$ \\
\hline Sexo & Hombres & 250 & 56.1 \\
Edad & Mujeres & 195 & 43.9 \\
& $<30$ & 21 & 4.8 \\
& $30-40$ & 81 & 18.2 \\
& $41-50$ & 230 & 51.6 \\
& $51-60$ & 93 & 21 \\
Categoría Profesional & $61-65$ & 17 & 3.7 \\
& $>65$ & 3 & 0.7 \\
& Catedráticos U. & 39 & 8.7 \\
& Titulares U./C.E.U & 261 & 58.6 \\
& T.E.U./Contrat.Dr. & 68 & 15.3 \\
Rama Conocimiento & Ayte.Doctor/Ayte. & 24 & 5.5 \\
& Asociado/Colabor. & 44 & 9.8 \\
& Otros & 9 & 2.1 \\
& Arte y Human. & 32 & 7.2 \\
& Ciencias & 43 & 9.5 \\
& CC. Salud & 77 & 17.3 \\
& CC.Soc y Jur. & 190 & 42.8 \\
& Arquit/Ingen. & 103 & 23.2 \\
\hline
\end{tabular}

Fuente: elaboración propia.

de sexo. Los estratos formaron a partir de las cinco Áreas de Conocimiento: Arte y Humanidades, Ciencias, Ciencias de la Salud, Ciencias Sociales y Jurídicas y Arquitectura e Ingenierías. Los cuestionarios se aplicaron mediante entrevista personal en los despachos de los profesores, o de manera autoadministrada con entrega y recogida concertada, mediante cita personal. El error muestral fijado para un nivel de confianza del $95.5 \%$ (dos sigmas) y $\mathrm{P}=\mathrm{Q}$ es de $\pm 4.32 \%$ para el conjunto de la muestra y en el supuesto de muestreo aleatorio simple. Además de estos, se realizó una afijación proporcional (por universidades) y ponderación mediante factor de elevación, una vez obtenida la muestra de universidades. El tamaño muestral asciende a 486 profesores universitarios en activo de las universidades públicas de Castilla y León (Salamanca, Valladolid, Burgos y León) sobre un total poblacional de 7.500 profesores. El trabajo de campo se efectuó en marzo y abril de 2012 (Tabla 1).

\section{Instrumentos}

Se diseñó y aplicó un cuestionario estructurado en dos partes, una sobre datos de identificación y características sociodemográficas y otra compuesta por una batería de 29 ítems o indicadores, basados en la revisión bibliográfica del marco teórico previo para la medida de las variables latentes del modelo propuesto. Todos ellas medidas con escalas tipo Likert graduadas desde muy probable a nada probable. Los ítems empleados como indicadores de la utilidad percibida se orientan a conocer las evaluaciones de los profesores acerca de las consecuencias que puede tener en la productividad de su actividad docente el uso de un determinado sistema de innovación (en este caso la intención de implementar la modalidad b-learning). Los indicadores de la escala facilidad de uso percibida valoran expectativas de resultados relacionados con la flexibilidad, el control o la sencillez a la hora de entender y utilizar esta modalidad formativa (complejidad vs. facilidad). 
Los indicadores de Imagen Social valoran el grado en el cual el uso de una innovación se percibe que aumenta la imagen o estatus de un sujeto en un sistema social (contexto universitario). La Facilidad de Condiciones (o también Compatibilidad) se refiere al grado en el que se percibe la existencia de infraestructura organizacional y técnica para dar soporte a la innovación.

Para la medida de la Norma Social se utilizó una escala tipo Likert de 4 puntos en la que se valoraba la percepción de los profesores sobre si otros creen que deberían usar o no el nuevo sistema. Para el diseño de esta escala, se realizó un estudio piloto previo en el que se identificaron tres tipos de categorías (creencias normativas): responsables del gobierno de la universidad (rectorado, decanato, dirección de departamento); compañeros (de centro y/o departamento, de grupo de investigación o de área de conocimiento); alumnos y otros. Por último, la intención conductual fue obtenida a partir de las respuestas dadas por los sujetos a dos ítems (IC en el semestre actual y en el próximo curso). Este tipo de escalas son las recomendadas por Ajzen y Fishbein (Ajzen, 2002b; Ajzen \& Fishbein, 1980), además de que existe evidencia substancial que manifiesta sus altos niveles de fiabilidad y validez (Ajzen \& Fishbein, 1980; Bagozzi, 2007).

\section{Resultados}

\section{Ajuste del modelo de medida: fiabilidad y validez de las escalas}

El análisis y depuración de datos implicó la identificación de casos atípicos (outliers) así como el análisis del impacto potencial de los datos ausentes (missing) sobre la representatividad de los datos. A partir de ahí y para garantizar la consistencia interna y la unidimensionalidad de las escalas, se comenzó con un análisis factorial exploratorio (AFE) a través del método de componentes principales, aplicando el criterio de Kaiser para cada constructo (es decir que solo para el primer componente principal el valor propio es mayor que 1). Se comprueba que los indicadores que integran cada constructo son unidimensionales. Para evaluar la homogeneidad de las escalas de medida de cada una de las variables que aparecen en el modelo, se utilizó la correlación de los ítems con el total de la escala y el coeficiente alfa de Cronbach. A partir de ahí, se eliminaron los ítems presentes en los factores para garantizar valores de alpha iguales o superiores a 0.7 (mínimo establecido). De igual modo, para la correlación ítem-total, se fijó el criterio de que todos los casos debían obtener valores por encima del mínimo exigido (> 0.3; Nurosis, 1993). En la segunda fase de validación de los constructos propuestos, se efectuó un análisis factorial confirmatorio (AFC) conforme al enfoque de ecuaciones estructurales (SEM) con estimación de parámetros, utilizando el método de Máxima Verosimilitud (ML), por reunir, entre otras características, las de ser invariante al tipo de escala y normalmente distribuido si las variables observables responden a las condiciones de normalidad. Se utilizó para ello el programa estadístico AMOS versión 16.0. Se analizó un modelo de medida en el que las diferentes variables latentes correlacionaban libremente. El modelo fue identificado ya que cada variable latente tenía al menos dos indicadores (Bollen \& Long, 1993; McDonald \& Ho, 2002). Los resultados del AFC muestran que el modelo de medida inicial presenta 56 variables, de las cuales 31 son variables exógenas y 25 , variables endógenas; aparecen 25 variables observadas o indicadores. Junto a ellas se aprecian asociados los errores de medida y los 6 constructos o variables latentes. Para asegurar la validez de los posteriores contrastes, se examinó la normalidad univariada y multivariada como condición necesaria para el uso del método de Maxima Verosimilitud (ML). Para ello, se aplicó como prueba el contraste de Kolmogorov-Smirnov con resultados satisfactorios. Por otro lado, a partir del programa AMOS se obtuvieron los valores absolutos de asimetría y curtosis. Se decidió eliminar aquellos ítems con valores superiores a 2 (West, Finch \& Curan, 1995). Los resultados (Tabla 2) mostraron normalidad univariada de los datos, así como la existencia de normalidad multivariada, ya que el coeficiente de Mardia obtenido 140.75 (curtosis multivariada de 40.4) es inferior a $p^{*}(p+2)=$ 675 (siendo $p$ el número de variables observadas) (Bollen, 1989; Bollen \& Long, 1993). 
TABLA 2

Assessment of normality (Group number 1)

\begin{tabular}{|c|c|c|c|c|}
\hline Variable & skew & c.r. & kurtosis & c.r. \\
\hline IC. 2 & -1.404 & -12.087 & 1.267 & 5.456 \\
\hline IC.1 & -1.107 & -9.537 & 0.379 & 1.631 \\
\hline NS.3 & 0.187 & 1.613 & -0.6 & -2.585 \\
\hline NS2 & -0.27 & -2.324 & 0.706 & 3.04 \\
\hline NS1 & 0.029 & 0.251 & -0.108 & -0.464 \\
\hline ISO.1 & 0.115 & 0.986 & -0.83 & -3.576 \\
\hline ISO.2 & -0.565 & -4.866 & -0.038 & -0.162 \\
\hline ISO.3 & -0.332 & -2.862 & -0.792 & -3.409 \\
\hline FC.1 & -1.257 & -10.823 & 1.326 & 5.712 \\
\hline FC.2 & -0.932 & -8.03 & 0.276 & 1.189 \\
\hline FC. 3 & -0.754 & -6.489 & 0.063 & 0.271 \\
\hline FUP.1 & -0.787 & -6.781 & -0.125 & -0.536 \\
\hline FUP.2 & -0.840 & -7.236 & 0.274 & 1.18 \\
\hline FUP.3 & -0.731 & -6.297 & -0.081 & -0.351 \\
\hline FUP.4 & -0.749 & -6.447 & 0.045 & 0.194 \\
\hline UP.1 & -0.298 & -2.564 & -0.296 & -1.276 \\
\hline UP.2 & -0.368 & -3.169 & -0.141 & -0.607 \\
\hline UP.3 & -0.617 & -5.31 & 0.149 & 0.643 \\
\hline UP.4 & -0.497 & -4.281 & -0.056 & -0.241 \\
\hline UP.5 & 0.014 & 0.122 & -0.538 & -2.318 \\
\hline UP.6 & -0.574 & -4.939 & 0.319 & 1.375 \\
\hline UP.7 & -0.117 & -1.011 & -0.415 & -1.788 \\
\hline UP.8 & -0.556 & -4.789 & 0.22 & 0.949 \\
\hline UP.9 & -0.633 & -5.448 & 0.168 & 0.724 \\
\hline UP.10 & -0.084 & -0.723 & -0.308 & -1.328 \\
\hline Multivariate & & 140.749 & 40.404 & \\
\hline
\end{tabular}

Fuente: elaboración propia.

Como medidas de consistencia interna de los indicadores de cada factor se calculó el índice de Fiabilidad Compuesta (FCC), tomando como criterio el valor recomendado superior a 0.7 (Lévy \& Varela, 2006), así como la cantidad total de la varianza de los indicadores tenida en cuenta por cada constructo latente (Average Variance Extracted), cuyo valor recomendable debe ser superior a 0.5 (Baggozi \& Yi, 1998). En el presente caso, únicamente la variable latente ISO no cumple este criterio por muy poco (0.48), por lo que se decide mantenerla en el modelo. Por su parte, la validez de las escalas del modelo se planteó a partir de la validez de contenido y la validez de constructo. La primera viene avalada por la revisión bibliográfica efectuada y sintetizada en el anterior epígrafe, y que ha servido para definir los conceptos contrastados en el estudio, mientras que para comprobar la validez de constructo se analizaron la validez convergente y la discriminante. Respecto a la validez convergente se observó que todos los ítems son significativos a un nivel de confianza de 95\% (imponiendo la máxima exigencia para el valor del estadístico $t$ de Student $t>2.58$; $p=$ 0.01 ). El cumplimiento de este requisito garantizaba que todos los indicadores estuvieran relacionados significativamente con su correspondiente variable latente, así como sus coeficientes lambda estandarizados $(\lambda)$ superiores a 0.5 (condición de convergencia fuerte) (Kline, 2005).

La validez discriminante implica que cada constructo debe ser significativamente diferente del resto de los constructos con los que no se 
TABLA 3

Modelo de medida: covarianzas significativas y relación entre las variables latentes

\begin{tabular}{|c|c|c|c|c|c|c|c|}
\hline \multicolumn{2}{|c|}{$\mathrm{N}$} & UP & FUP & CF & ISO & NS & IC \\
\hline UP & 445 & 0.81 & $0.176^{* * *}$ & $0.274 * *$ & $0.655 * *$ & $0.422 * *$ & $0.557^{* *}$ \\
\hline FUP & 445 & $0.176^{* *}$ & 0.81 & $0.468^{* *}$ & -0.031 & $0.107^{*}$ & $0.259 * *$ \\
\hline $\mathrm{CF}$ & 445 & $0.274^{* *}$ & $0.468^{* * *}$ & 0.77 & $0.182 * *$ & $0.186^{* *}$ & $0.362 * *$ \\
\hline ISO & 445 & $0.655^{* * *}$ & -0.031 & $0.182 * *$ & 0.69 & $0.397^{* *}$ & $0.427^{* *}$ \\
\hline NS & 445 & $0.422 * *$ & $0.107 *$ & $0.186^{* *}$ & $0.397 * *$ & 0.82 & $0.352 * *$ \\
\hline IC & $\mathrm{r}$ & $0.557^{* *}$ & $0.259 * *$ & $0.362 * *$ & $0.427 * *$ & $0.352 * *$ & 0.93 \\
\hline
\end{tabular}

*. La correlación es significante al nivel 0.05 (bilateral).

**. La correlación es significativa al nivel 0.01 (bilateral).

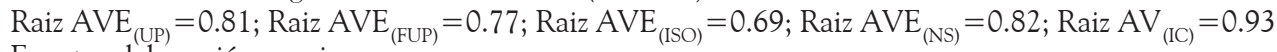

Fuente: elaboración propia.

encuentra relacionado, según la teoría. Para analizar la validez discriminante se obtuvo la matriz de cargas factoriales y de cargas factoriales cruzadas (coeficientes de correlación de Pearson entre los indicadores y los otros constructos), comprobando que los indicadores estuvieron más correlacionados con su propio constructo que con los otros. De otro modo, se comprobó que la raíz cuadrada del AVE del constructo fuera mayor que la correlación entre ese constructo y todos los demás (Chin, 1998), evidenciando además que las correlaciones entre los distintos factores que componen el modelo no fueran superiores a 0.8 (Bagozzi, 1994). La Tabla 3 muestra los coeficientes de correlación entre los constructos, así como el valor de la raíz cuadrada de AVE (en negrita) para el estudio de la validez discriminante.

Como puede observarse (Tabla 4), las medidas de bondad de ajuste muestran valores que están dentro de los parámetros recomendados.

\section{Análisis del modelo estructural y contraste de las hipótesis del estudio}

Una vez analizado y obtenidos los resultados del modelo estructural observamos (Tabla 5) que la mayoría de los indicadores de ajuste alcanzan los valores óptimos recomendados (valores superiores a 0.9 son considerados como aceptables; Bentler, 1995). En particular destacamos como medidas más representativas de ajuste absoluto la ratio entre chi cuadrado y los grados de libertad $\left(\chi^{2} / g l\right)$ (utilizamos este heurístico en lugar del $\chi^{2}$ para reducir la sensibilidad de éste al tamaño de la muestra); el GFI: (Goodness of Fit Index) y NFI (Normed Fit Index) como medidas de discrepancia entre el modelo ajustado y el modelo base. Asimismo, el RMSEA (Root Mean Square Error Aproximation) como un índice que proporciona una medida de discrepancia por grado de libertad). Asimismo, se consideraron como indicadores de ajuste incremental el CFI (Comparative Fit Índex) por ser uno de los indicadores más importantes, preferible a chi cuadrado para muestras superiores a 200 casos y otros indicadores muy consistentes como son el TLI (Índice de Tucker) e IFI.

A la vista de los resultados (Tabla 5), se puede afirmar que el modelo estructural resulta adecuado para contrastar las hipótesis planteadas en este trabajo, asumiendo como condición de significación los coeficientes path estandarizados, los valores de la $t$ de Student y los $R^{2}$ (R-square).

\section{Discusión y conclusiones}

La literatura que analiza los elementos o variables que intervienen en la adopción de una innovación tanto a nivel externo como interno identifica una de constructos que establecen una relación entre ellos para explicar la decisión final del individuo a la hora de adoptar o usar esa innovación. Bajo esta perspectiva, se entiende que si se puede identificar el o los factores que favorecen o bien desfavorecen la acción de adopción de una innovación dada, 
TABLA 4

Resultados del Análisis Factorial Confirmatorios

\begin{tabular}{|c|c|c|c|c|c|c|}
\hline $\begin{array}{l}\text { Variable } \\
\text { Latente }\end{array}$ & $\begin{array}{l}\text { Variable } \\
\text { Observada }\end{array}$ & $\begin{array}{l}(\lambda) \text { Lambda } \\
\text { Estand. }>0.50\end{array}$ & $\begin{array}{c}\mathrm{R}^{2} \text { Squared Multiple } \\
\text { Correlations }\end{array}$ & $\begin{array}{l}\alpha \text { alpha } \\
\text { Cronbach }\end{array}$ & $\begin{array}{l}\text { FCC Fiabilidad } \\
\text { Compuesta }\end{array}$ & AVE \\
\hline \multirow{10}{*}{$\begin{array}{l}\text { Utilidad } \\
\text { percibida }\end{array}$} & UP1 & 0.79 & 0.62 & 0.94 & 0.91 & 0.65 \\
\hline & UP2 & 0.85 & 0.72 & & & \\
\hline & UP3 & 0.83 & 0.71 & & & \\
\hline & UP4 & 0.84 & 0.7 & & & \\
\hline & UP5 & 0.73 & 0.53 & & & \\
\hline & UP6 & 0.77 & 0.59 & & & \\
\hline & UP7 & 0.79 & 0.62 & & & \\
\hline & UP8 & 0.80 & 0.63 & & & \\
\hline & UP9 & 0.70 & 0.48 & & & \\
\hline & UP10 & 0.66 & 0.43 & & & \\
\hline \multirow{4}{*}{$\begin{array}{l}\text { Facilidad de } \\
\text { uso percibida }\end{array}$} & FUP1 & 0.64 & 0.41 & 0.87 & 0.88 & 0.66 \\
\hline & FUP2 & 0.95 & 0.89 & & & \\
\hline & FUP3 & 0.9 & 0.8 & & & \\
\hline & FUP4 & 0.71 & 0.51 & & & \\
\hline \multirow{3}{*}{$\begin{array}{l}\text { Condiciones } \\
\text { facilitador }\end{array}$} & $\mathrm{CF} 1$ & 0.72 & 0.52 & 0.8 & 0.81 & 0.59 \\
\hline & $\mathrm{CF} 2$ & 0.86 & 0.74 & & & \\
\hline & $\mathrm{CF} 3$ & 0.72 & 0.51 & & & \\
\hline \multirow[t]{3}{*}{ Image social } & ISO1 & 0.69 & 0.41 & 0.73 & 0.74 & 0.48 \\
\hline & ISO2 & 0.64 & 0.47 & & & \\
\hline & ISO3 & 0.75 & 0.56 & & & \\
\hline \multirow{3}{*}{$\begin{array}{l}\text { Influencia } \\
\text { social }\end{array}$} & NS1 & 0.93 & 0.87 & 0.85 & 0.86 & 0.68 \\
\hline & NS2 & 0.58 & 0.34 & & & \\
\hline & NS3 & 0.92 & 0.85 & & & \\
\hline \multirow{2}{*}{$\begin{array}{l}\text { Iintención de } \\
\text { uso bl }\end{array}$} & IC1 & 0.94 & 0.88 & 0.93 & 0.93 & 0.87 \\
\hline & $\mathrm{IC} 2$ & 0.93 & 0.86 & & & \\
\hline $\begin{array}{l}\text { Indices de } \\
\text { ajuste }\end{array}$ & $\begin{array}{l}\chi 2=659.2 \\
R M R=0.04\end{array}$ & $\begin{array}{l}0.00000) ; \chi^{2} / \mathrm{g} \\
\text { MSEA }=0.059\end{array}$ & $2.53 ; \mathrm{CFI}=0.94 ; \mathrm{TL}$ & $4 ; \mathrm{NFI}=$ & $\mathrm{IFI}=0.94 ; \mathrm{PC}$ & $=0.88$; \\
\hline
\end{tabular}

Fuente: elaboración propia.

TABLA 5

Índices de bondad de ajuste del modelo causal

\begin{tabular}{cccc}
\hline Medidas absolutas de ajuste & Valor & Medidas de Ajuste Incremental & Valor \\
\hline$\chi 2 / D F$ & 2.25 & NFI & 0.91 \\
GFI & 0.9 & IFI & 0.95 \\
NFI & 0.91 & TLI & 0.94 \\
RMR & 0.045 & CFI robusto & 0.95 \\
RMSEA & 0.053 & & \\
& & & \\
\hline HOLTER & $>200$ & $226 / 239$ & \\
\hline
\end{tabular}

Fuente: elaboración propia.

de un sujeto o grupo, es posible tomar decisiones que conduzcan a impulsar un mayor o mejor uso de esa innovación. En este caso, se ha tratado de testar empíricamente un modelo TAM adaptado al 
TABLA 6

Resumen prueba de hipótesis

\begin{tabular}{|c|c|c|c|c|c|}
\hline Hipótesis & Relación & $\beta$ Coeficiente estandarizado & $\mathrm{p}$ & Valor t robusto & CONTRASTE \\
\hline $\mathrm{H} 1$ & UP IC & $\beta=0.56$ & $* * *$ & & Aceptada \\
\hline $\mathrm{H} 2$ & FUP IC & & $<0.05$ & & Rechazada \\
\hline $\mathrm{H} 3$ & CF IC & $\beta=0.26$ & $* * *$ & & Aceptada \\
\hline $\mathrm{H} 4$ & NS UP & & $<0.05$ & & Rechazada \\
\hline $\mathrm{H} 5$ & ISO UP & $\beta=0.84$ & $* * *$ & & Aceptada \\
\hline $\mathrm{H} 6$ & FUP UP & $\beta=0.2$ & $* * *$ & & Aceptada \\
\hline $\mathrm{H} 7$ & CF FUP & $\beta=0.48$ & $* * *$ & & Aceptada \\
\hline $\mathrm{H} 8$ & NS ISO & $\beta=0.69$ & $* * *$ & & Aceptada \\
\hline
\end{tabular}

$* * * p<0.001 * * *<0.005$.

Fuente: elaboración propia.

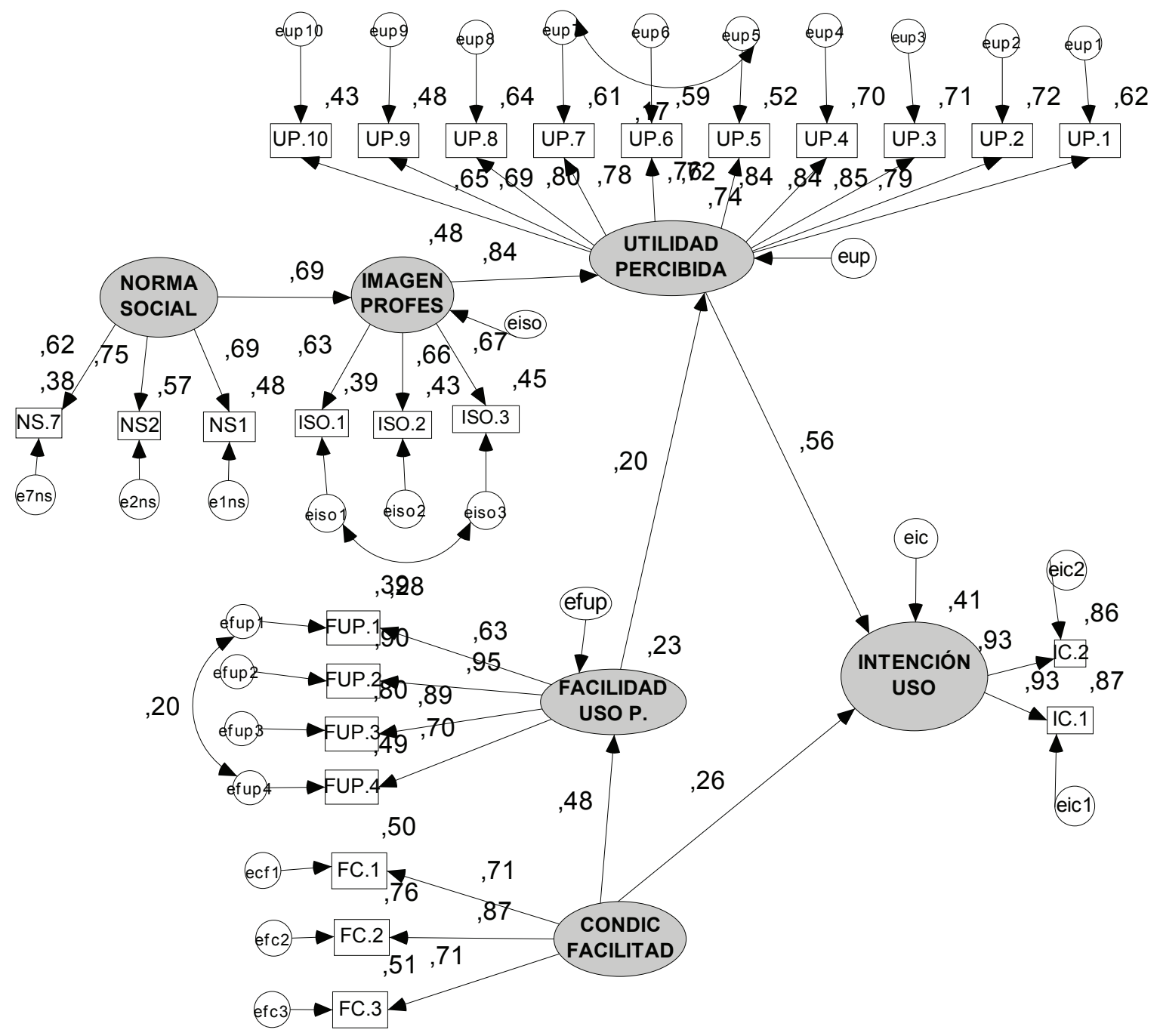

Gráfico2. Modelo empírico obtenido.

Fuente: elaboración propia. 
uso de la modalidad formativa blended learning en contextos universitarios, con el objetivo de proporcionar una explicación de los determinantes de la aceptación de uso de esa modalidad formativa. Una vez validado, el total de la varianza explicada por el modelo probado es del $41 \%\left(R^{2}=0.41\right)$, lo cual está en consonancia con otros resultados contenidos en la literatura sobre el tema (en relación con la capacidad explicativa de los diferentes modelos Venkatesh y Davis [2000] señalan que la mayoría de trabajos alcanzan valores comprendidos entre el $20 \%$ y el $70 \%$ ). Esta variabilidad explicada de la intención de los profesores está condicionada fundamentalmente por dos factores o variables. De un lado, la percepción del sujeto sobre la utilidad que esta innovación puede tener en la mejora de su práctica docente $(\beta=0.54, p=0.00)$ y de otro, aunque en bastante menor medida, la existencia de condiciones favorables (recursos y equipamientos técnicos, humanos, etc. disponibles en la universidad) $(\beta=0.26, p=0.00)$. Ambos factores son lógicos en el contexto de la formación universitaria. Quizá, lo más llamativo es el escaso papel que ofrece la variable facilidad de uso sobre la intención, en contra de lo indicado en nuestro modelo teórico. Este resultado es interesante y muestra que, en un contexto altamente profesionalizado como es el universitario, la percepción de esfuerzo que puede conllevar la introducción de una innovación no es un condicionante importante a la hora de asumir o incorporar ese nuevo elemento docente. En realidad, este resultado corrobora la línea de estudios que avalan una escasa importancia de la facilidad de uso, al no presentar un impacto directo consistente en la fase de aceptación e incluso, como se ha comprobado, puede volverse no significativo en las decisiones de uso posteriores (Davis et al., 1989; Karahanna et al., 1999). Pero, aunque no de modo directo, este efecto sobre la intención sí aparece modulado por la utilidad percibida- que mediatiza también el efecto de la percepción de la imagen profesional del docente al adoptar la innovación $(\beta=0.48, p=0.001)$.

Algo similar ocurre con el papel de la influencia o norma social sobre la intención de uso. En el presente estudio no aparece relación causal significa- tiva entre esta variable y la intención de adopción, lo cual apoya resultados similares en el contexto del e-learning (Lee, 2006) y sí tiene un efecto indirecto sobre UP mediatizado por la Imagen Social (profesional). La idea más extendida en relación con esta variable es que su efecto es indirecto sobre la IP a través de la utilidad percibida y únicamente tiene un impacto significativo en situaciones en las que se exige a los sujetos el uso de un determinado sistema o innovación (Schepers \& Weltzels, 2007), cosa que no sucede en el entorno de las universidades públicas. Por otro lado, se encuentra que la utilidad percibida de usar o no la modalidad b-learning aparece explicada (en un $73 \%$ de la variabilidad) por dos tipos de efectos directos; de un lado, la imagen social $(b=0.83) y$, en bastante menor medida, la facilidad de uso percibida $(b=0.2)$. A su vez, la imagen social/profesional se ve explicada (69\%) por los referentes significativos para el sujeto (NS) y la facilidad de uso por la variable condiciones facilitadoras (48\%).

Por último, entre las limitaciones de este estudio hay que señalar que, aunque en la literatura existen numerosos estudios que utilizan TAM para evaluar la aceptacion de e-learning en estudiantes y profesores universitarios, sin embargo, son pocos aún los trabajos que analizan la aceptación de la modalidad b-learning con los que poder contrastar los resultados. Por otro lado, la principal limitación del presente estudio tiene que ver con el hecho de que el blended learning no surge del e-learning, sino desde la enseñanza tradicional. Esto significa que muchos profesores del estudio conocen esta modalidad formativa y, o bien no son plenos usuarios de la misma o bien trasladan parte de su rutina docente basada en la clase presencial, al formato e-learning, sin la necesaria integración equilibrada de ambas modalidades. Por este motivo, en la muestra de la investigación no se ha diferenciado entre adoptantes tempranos o adoptantes expertos, en la idea de dar una visión del conjunto del profesorado. Por ello, es deseable ampliar y desarrollar nuevos estudios en los que se diferencien claramente subgrupos, en función del nivel de uso y conocimiento de $b$-learning, permitiendo una mejora del modelo explicativo. 


\section{Referencias}

Ajzen, I. (1991). The theory of planned behavior. Organizational Behavior and Human Decision Processes, 50(2), 179-211.

Ajzen, I. \& Fishbein, M. (1980). Understanding attitudes and predicting social behaviour. Englewood Cliffs, NJ: Prentice-Hall.

Atkinson, M. \& Kydd, C. (1997). Individual characteristics associated with world wide web use: An empirical study of playfulness and motivation. The DATA BASE for Advances in Information Systems, 28(2), 53-62.

Bagozzi, R. P. (2007). The legacy of the technology acceptance model and a proposal for a paradigm shift. Journal of the Association for Information Systems, 8(4), 244-254.

Bagozzi, R. P., Davis, F. D. \& Warshaw, P. R. (1992). Development and test of a theory of technological learning and usage. Human Relations, 45(7), 660-686.

Bagozzi, R. P., Davis, F. D. \& Warshaw, P. R. (1992). Development and test of a theory of technological learning and usage. Human Relations, 45(7), 660-686.

Bagozzi, R., Yi, Y. \& Nassen, K.D. (1998). Representation of measurement error in marketing variables: Review of approaches and extension to three-facet designs. Journal of Econometrics, 89(1-2), 393-421.

Bandura, A. (1982). Self-efficacy mechanism in human agency. American Psychologist, 37(2), 122-147.

Bentler, P. (1995). EQS structural equations program manual. Encino, CA: Multivariate Software.

Bersin, J. (2004). The blended learning book: Best practices, proven methodologies, and lessons learned. San Francisco: Wiley.

Bollen K. A. (1989). Structural equations with latent variables. New York: Wiley.

Bollen, K. A. \& Long, J. S. (1993). Testins structural equation models. Newbury Park, CA: Sage.

Bonk, C. J. \& Graham, C. R. (Eds.). (2006). The handbook of blended learning: Global perspectives, local designs. San Francisco: Pfeiffer Publishing.

Chin, W. W. (1998). The partial least squares approach to structural equation modeling. En G. A. Mar- coulides (Ed.), Modern methods for business research (pp. 195-336). Mahwah, NJ: Erlbaum.

Davis, F. D. (1989). Perceived usefulness, perceived ease of use, and user acceptance of information technology. MIS Quarterly, 13(3), 319-340.

Davis, F. D., Bagozzi, R. P. \& Warshaw, P. R. (1989). User acceptance of computer technology: A comparison of two theoretical models. Management Science, 35(8), 982-1003.

Davis, F. D., Baggozzi, R. P. \& Warshaw, P. R. (1992). Extrinsic and intrinsic motivation to use computers in the workplace. Journal of Applied Social Psychology, 22(14), 1111-1132.

Fishbein, M. \& Ajzen, I. (1975). Belief, attitude, intention and behavior: An introduction to theory and research. Reading, MA; Addison-Wesley.

Garrison, D. R. G. \& Vaughan, N. D. (2008). Blended learning in higher education: Framework, principles and guidelines. San Francisco: Pfeiffer Publishing.

Gefen, D., Karahanna, E. \& Straub, D. (2003). Inexperience and experience with online stores: The importance of TAM and Trust. IEEE Transactions on Engineering. Management, 50(3), 307-321.

Gerbic, P. (2009). Effective blended learning practices: Evidence-based perspectives in ICT-Facilitated Education. Hershey, PA: IGI Global Snippet.

Lévy Mangin, J. \& Varela Mallou, J. (Eds.). (2006). Modelización con estructuras de covarianzas en ciencias sociales. Madrid: Netbiblo.

Igbaria, M., Livari, J. \& Maragahh, H. (1995). Why do individuals use computer technology? A finnish case study. Information $\mathcal{E}$ Management, 29(5), $227-238$

Karahanna, E., Agarwal, R. \& Angst, C. (2006). Reconceptualizing compatibility beliefs in technology acceptance research. MIS Quarterly, 30(4), 781-804.

Kitchenham, A. (2011). Blended learning across disciplines: Models for implementation. Hershey, PA: IGI Global Snippet.

Kline, B. R. (2005). Principles and practice of structural equation modeling (2nd ed.). New York: Guilford Press.

Lederer, A. L., Maupin, D. J., Sena, M. P. \& Zhuang, Y. (2000). The technology acceptance model and the world wide web. Decision Support Systems, 29(3), 269-282. 
Lee, Y. C. (2006). An empirical investigation into factors influencing the adoption of e-learning system. Online Information Review, 30(5), 517-541.

Lee, M. K. O., Cheung, C. M. K. \& Chen, Z. (2005). Acceptance of internet based learning medium: The role of extrinsic and intrinsic motivation. Information E Management, 42(8), 1095-1104.

Lin, W. \& Wang, Ch. (2012). Antecedences to continued intentions of adopting e-learning system in blended learning instruction: A contingency framework based on models of information system success and task-technology fit. Computers $\mathbb{E}$ Education, 58(1), 88-99.

Nurosis, M. (1993). SPSS. Statistical data analysis. Chicago, IL: SPSS Inc.

Ong, C., YuLai, J. \& Wang, Y. (2004). Factors affecting engineers' acceptance of asynchronous elearning system in high tech companies. Information $\mathbb{E}$ Management, 41(6), 795-804.

Rogers, E. (1995). Diffusion of innovations (4th ed.). New York: Free Press.

Rogers, E. (2009). Understanding technology adoption: Theory and future directions for informal learning. Review of Educational Research, 79(2), 625-649.

Schepers, J. \& Wetzels, M. (2007). A meta-analysis of the technology acceptance model: Investigating subjective norm and moderation effects. Information E Management, 44(1), 90103.

Selim, H. M. (2003). An empirical investigation of student acceptance of course websites. Computers $\mathbb{E}$ Education, 40(4), 343-360.
Taylor, S. \& Todd, P. (1995). Understanding information technology usage: A test of competing models. Information Systems Research, 6(2), 144-176.

Venkatesh, V. \& Bala, H. (2008). Technology acceptance model 3 and a research agenda on interventions. Decision Sciences, 39(2), 273-315.

Venkatesh, V. \& Davis, F. D. (2000). A theoretical extension of the technology acceptance model: Four longitudinal field studies. Management Science, 46(2), 186-204.

Venkatesh, V., Morris, M. G., Davis, G. B. \& Davis, F. D. (2003). User acceptance of information technology: Toward a unified view. MIS Quarterly, 27(3), 425-478.

Wang, F. L., Fong, J. \& Kwan, R. (2009). Handbook of research on hybrid learning models: Advanced tools, technologies and applications. Hershey, PA: IGI Global Snippet.

West, S. G., Finch, J. F. \& Curan, P. J. (1995). Structural equation models with nonnoral variables: Problems and remedies. En R. H. Hoyle (Ed.), Structural equations modelling: Concepts, issues, and applications (pp. 56-75). Thousand Oaks, CA: Sage Publications.

Yi, M. Y. \& Hwang, Y. (2003). Predicting the use of web based information systems: Selfefficacy, enjoyment, learning, goal orientation, and the technology acceptance model. International Journal Human Computer Studies, 59(4), 431-449. 